International Journal of Engineering \& Technology, $7(2.24)(2018) 102-105$
International Journal of Engineering \& Technology
SPC
Website: www.sciencepubco.com/index.php/IJET
Research paper

\title{
Angle and Scale Invariant Template Matching for Handling Image Distortions
}

\author{
Badrinaathan.J", L.N.B.Srinivas \\ ${ }^{1,2}$ SRM UNIVERSITY, Chennai,India \\ *Corresponding Author Email: badrinaathan_jagadeesan@srmuniv.edu.in
}

\begin{abstract}
Template matching is a diagnostic approach for detecting a patch of a template image in a given source image. This plays a vital role in multitudinal computer vision applications. In this paper, we propose a methodology that makes the naive template matching algorithm scale and angle invariant during the image recognition process where the source and template is converted to gray scale which makes the technique enhance its proficiency. The proposed algorithm handles the arbitrary modulations of the image patch with respect to size and angle by an exhaustive search of all combinations of sizes are done along with populous combinations of angles. The images adapted are subjected to certain filtering and convolution methods which deepens the quality of the images which in turn assists in retrieving the features with accuracy. The image intensities are adjusted using histogram equalization to enhance the image contrast. These images are then subjected to perform template matching using normalized cross correlation to measure similarity between those two images.
\end{abstract}

Key words: Template Matching, Filtering, Scale Invariant, Histogram Equalization, Angle Invariant, Cross Correlation

\section{Introduction}

Template matching is a technique in the field of image processing to identify small patches of an image in a reference image. Identifying the best matching location in a reference image is a challenging problem.

The template matching algorithm that is used now is insufficient for real time applications. This algorithm performs matching by positioning the template patch given over the source image in every possible region and we will obtain the measure of similarity between the template and the source image that overlaps with. When the template image given is different from the reference image in orientations the accuracy in identification becomes less. Under these assumptions, this paper considers comparing two images invariant to size and angle, which recognizes the image patch (template image) in the source image.

To identify the matching locations using match template function, certain features of the image has to be extracted. One of the important features is considered to be the edges of the image, obtained through approaches like search-based and zero-crossing based edge detection. Edges can be defined as the pixels points in an image where the image brightness can vary and has certain discontinuities which helps in comparison. Here, the solution for handling image distortions is provided by multiple searches for all combinations of sizes and rotation is carried out. The thumb rule here is to extract some statistical features of the template image and identify whether these features appear or present in the reference image. If identified the maximum and minimum value of location is obtained and visualized by bounding the location inside a rectangle. The algorithms applicability is limited mostly by the availability of computational power, as identification of big and complex templates are time-consuming.

\section{Related Works}

\section{a. Randomness Normalized Cross Correlation:}

With a specific end goal to diminish the computational multifaceted nature of layout coordinating, the versatile Randomness Normalized Cross Correlation (RNCC) is proposed. The new approach that is proposed relies upon irregular pixels for calculation, rather than dissecting each pixel in I. Arbitrariness Normalized Cross Correlation (RNCC) utilizes a gathering of pictures $I(i, j)$, comparing to irregular pixels that are chosen $I(i, j)$, noted as Random Pixel (RP), to register the NCC esteem amongst $\mathrm{T}$ and itself.

\section{b. Best-Buddies Similarity:}

Best-Buddies Similarity (BBS) depends on distinguishing the quantity of Best-Buddies Pairs (i.e.) sets of focuses in source and target sets, where each point is the closest neighbor of the other. Best-Buddies Similarity (BBS), dissects the key highlights, and perform broad assessment in view of its execution. To apply best amigo likeness to layout coordinating, we have to change over each picture fix to an arrangement of focuses in $\mathrm{Rd}$, which requires registering the separation between each combine of focuses.

\section{c. Buried Object Discrimination In Gpr Data:}

A format coordinating way to deal with separate the covered question is proposed over the ground entering radar (GPR) B-filter pictures. This approach is scale invariant, which adjusts for the adjustments in the swinging pace of the finder. Ground Penetration Radar signs can be spoken to in a few ways. Here the layout coordinating is done on binarized pictures to accelerate the 
calculation. Once the pictures are binarized, an effective likeness measure approximating the relationship for these twofold pictures is misused. To give scale invariance, a changed variant of Gaussian pyramid is utilized.

\section{Modeling and Methodology}

The aim in this paper is to match the template image with the source image given irrespective of the orientation of the template image. The methodology of this explained below;

Loading and processing image: The reference image and a patch that is present in the reference image are loaded using the library open-cv. Once the images are loaded they are converted to gray scale. The gray scale converts multi dimensional image to a one dimension array of pixel values. The gray scale image is subjected to image filtering to process the noises and enhance the image that makes the feature extraction process easier.

Image Filtering: Both the images are subjected to convolution to improve the quality of the image. In general the image convolution is an element wise matrix multiplication with a kernel matrix. Convolution, which is a fundamental building block in computer vision, can also be termed as image smoothing to ignore the noises that are present. Each element of the pixel matrix is added to the neighbors and then weighted by the kernel matrix as represented below;

$$
\mathrm{h}=\mathrm{x}[(\mathrm{i}+\mathrm{k}),(\mathrm{j}+\mathrm{l})], \quad \mathrm{w}=\mathrm{y}[(\mathrm{H}+\mathrm{k}),(\mathrm{W}+\mathrm{l})] \quad \operatorname{add}=(\mathrm{w} * \mathrm{a})
$$

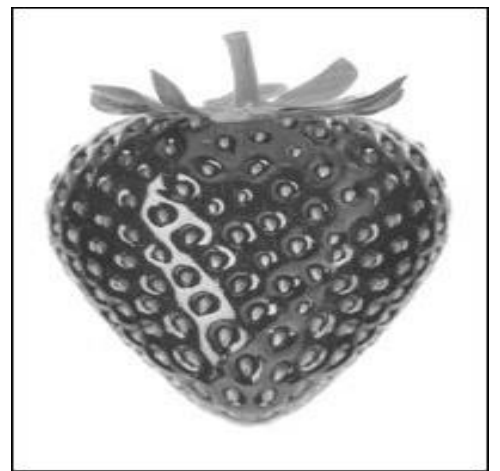

Fig. 1: template image after convolution

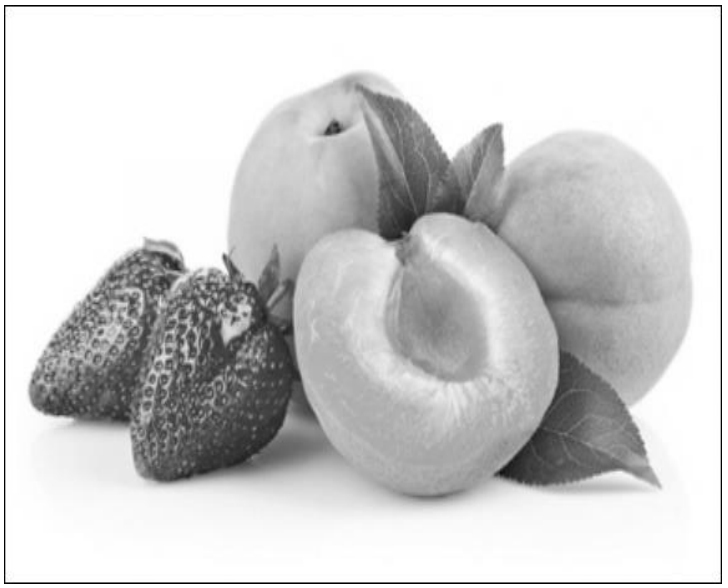

Fig. 2: source image after convolution

where $\mathrm{i}, \mathrm{j}, \mathrm{k}$ represents the pixel values multiplies with the kernel matrix and $\mathrm{H}, \mathrm{W}$ is the height and width of the image matrix.

Histogram and histogram equalization: The histogram of the reference image and the template image gives the plot of intensity of the image to its frequency. This plot helps to understand the distribution of pixel values both the images. Histogram equalization is to improve the contrast of the image, on which the quality of image is improved and process of feature extraction like edge detection is made less complicate.

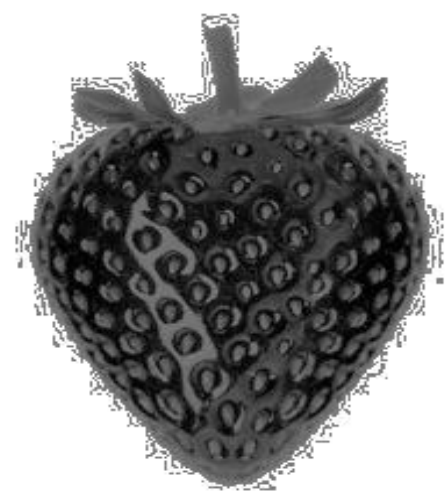

Fig. 3: Histogram Equalization of template image.

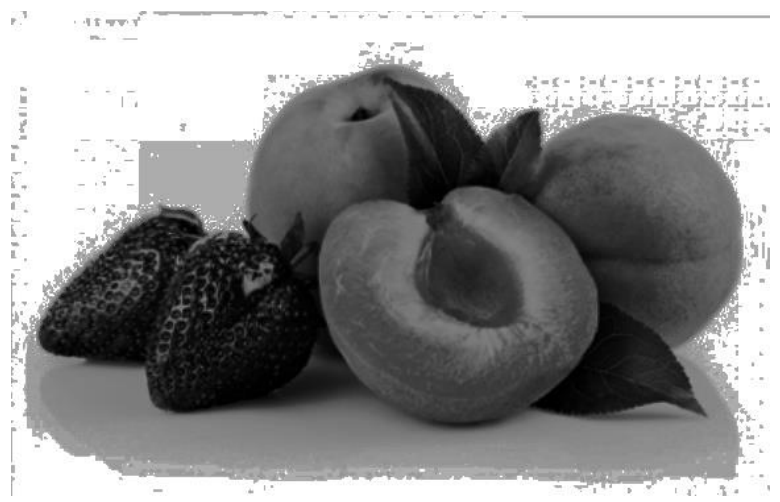

Fig. 4: source image after histogram equalization

Once the image enhancement is done, edges of both the images are extracted for template matching process.

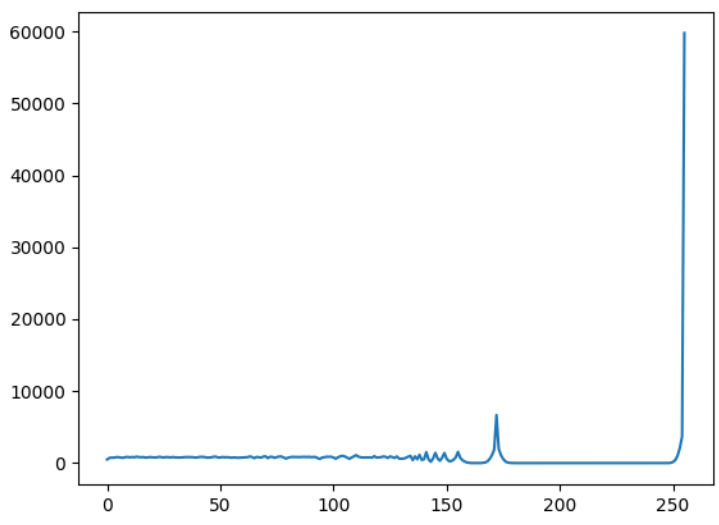

Fig. 5: Histogram of source image

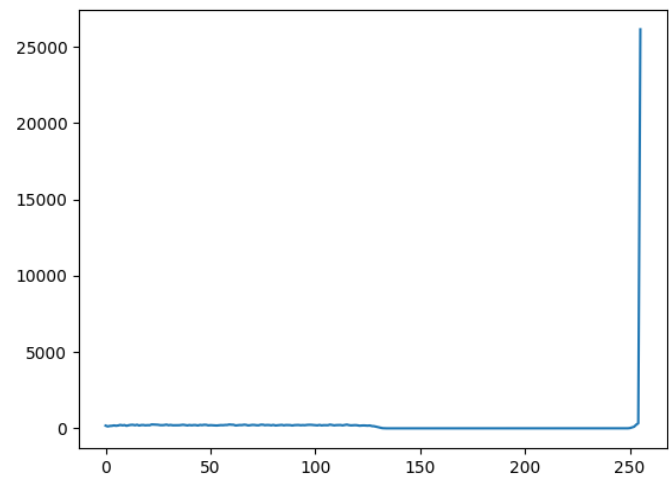

Fig. 6: Histogram of template image 


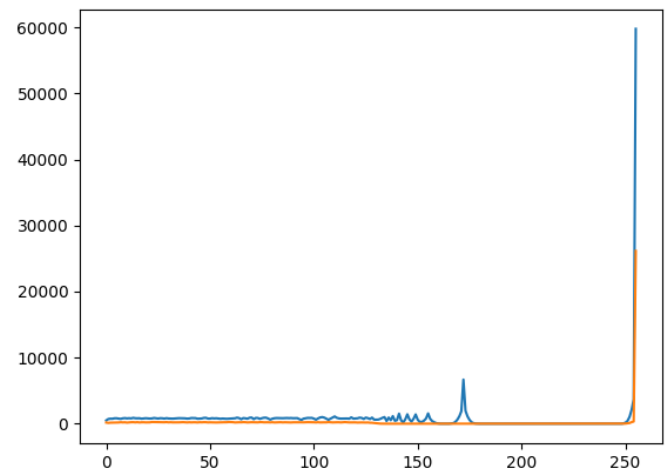

Fig. 7: Comparison of source and template image

Cross correlation: Finding the similarity between the template and the reference is one of the sub problems that occur during template matching. The similarity measure is obtained by finding the cross correlation between the two images. There might be multiple occurrence of the patch image in the source image. To obtain those locations the thresholding concept is used.

\section{Cross-correlation, $\mathrm{C}_{\mathrm{r}}=\mathrm{N} \Sigma \mathrm{xy}(\Sigma \mathrm{x})(\Sigma \mathrm{y}) / \sqrt{[}\left[\mathrm{N} \Sigma \mathrm{x}^{2}(\Sigma \mathrm{x})^{2}\right]\left[\mathrm{N} \Sigma \mathrm{y}^{2}-\right.$ $\left.(\Sigma y)^{2}\right]$}

Angle Invariance Model: To make the process of template matching angle invariant the images is subjected to matching process with various angles. The center of the template image is calculated and rotated keeping center as its axis of rotation. The angles for rotation are calculated using;

$\Omega_{\mathrm{y}}=-\sin (\pi) *(\mathrm{x}-$ TemplateWidth $)+\cos (\pi) *(\mathrm{y}-$ TemplateHeight $)+$

TemplateHeight / 2;

$\Omega_{\mathrm{x}}=\cos (\pi) *(\mathrm{x}-$ TemplateWidth $)+\sin (\pi) *(\mathrm{y}-$

TemplateHeight)+TemplateWidth / 2;

The rotation matrix obtained results in multiple angles of the template image as shown below;

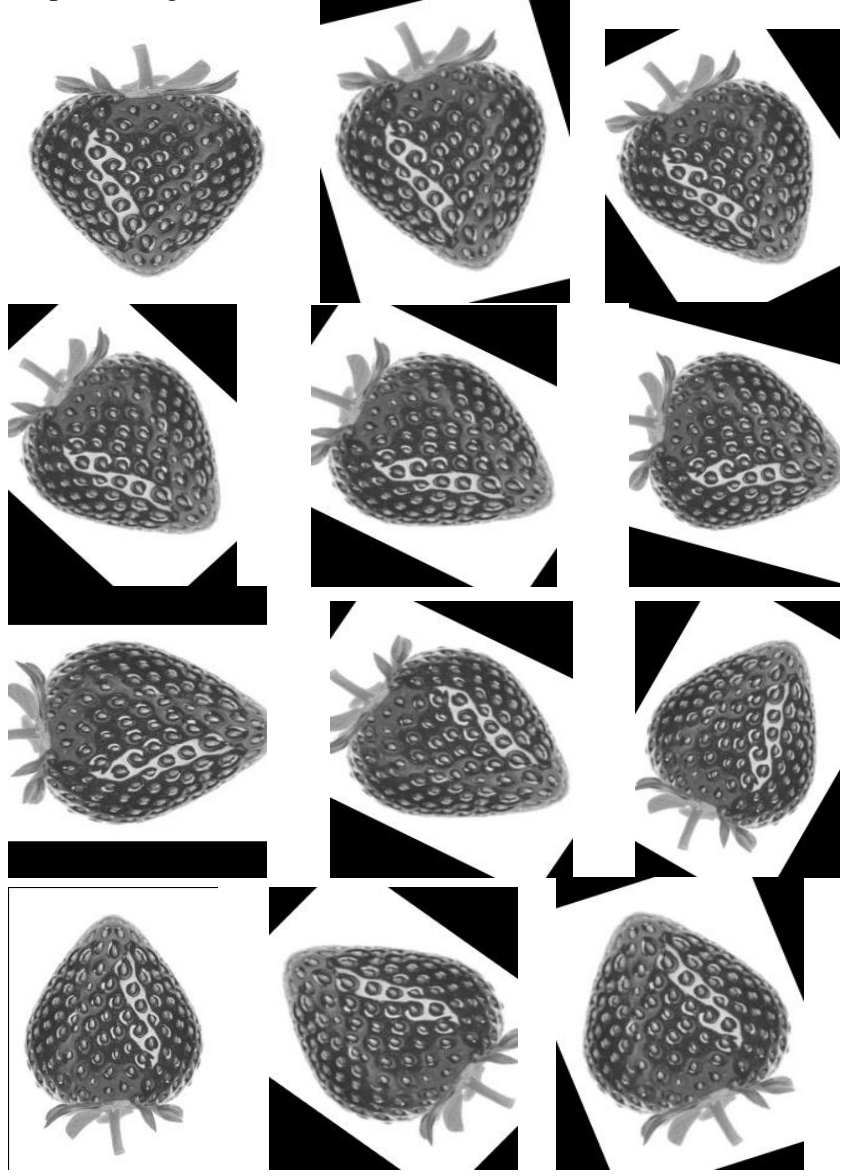

Fig. 5: Rotated template image with multiple angles.
Scale Invariant Model: On having the patch of template image in the reference image which is of different size, this algorithm inherits the scale invariant model to switch the template image to various sizes. The template matching performs matching process with exhaustive search of matching location with resized template image.
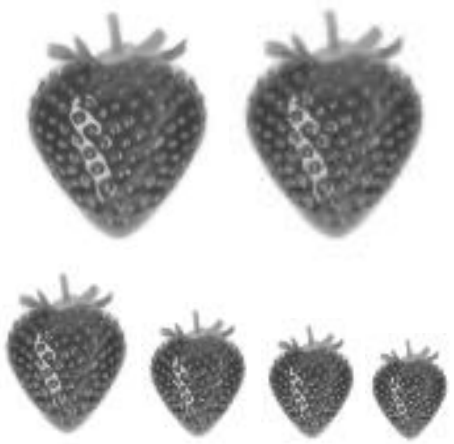

Fig. 6: Scaled template image

During image scaling one of the important tasks is to maintain the quality of the image. This is achieved by interpolation technique. Image interpolation is the method for identifying intensity values at locations where the pixel values are missing in an image. Interpolation constructs new data points with the known set of pixel values, where the known pixels from the original image can be used to obtain an interpolated image based on a simpler method. A major contribution is to greatly simplify occlusion handling during template matching. But few errors are usually present, when a interpolation technique is used during estimation of pixels from the original. The image quality highly depends on the used interpolation technique.

\section{Experimental Results}

In this section the results of the algorithm proposed is shown. The dimension of the template image used is $178 \times 90$ and of size $25 \mathrm{~KB}$. The dimension of the reference image used is $385 \times 451$ and of size $44.2 \mathrm{~KB}$. The images are downloaded from Google images. ]

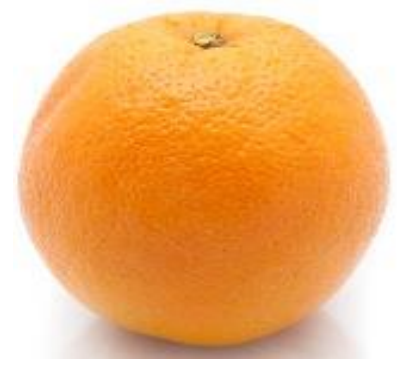

Fig. 8: Template image

[i] Image

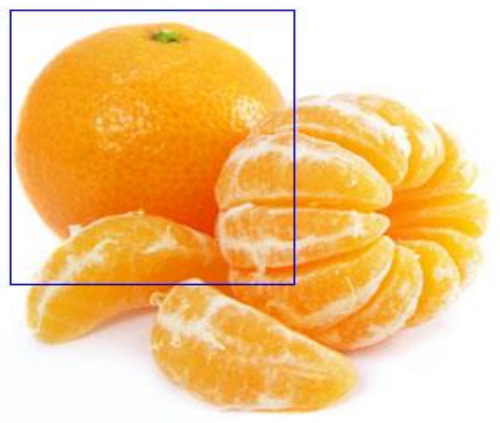

Fig. 9: Source image 


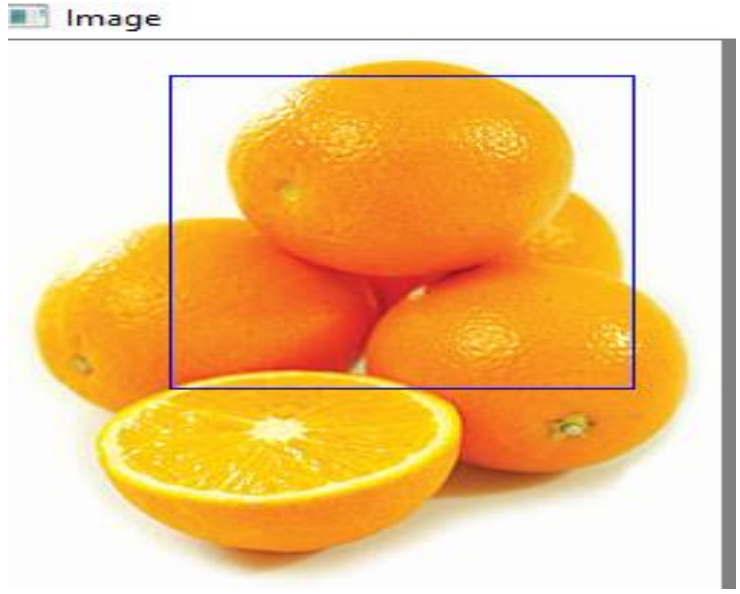

Fig. 10: Source image

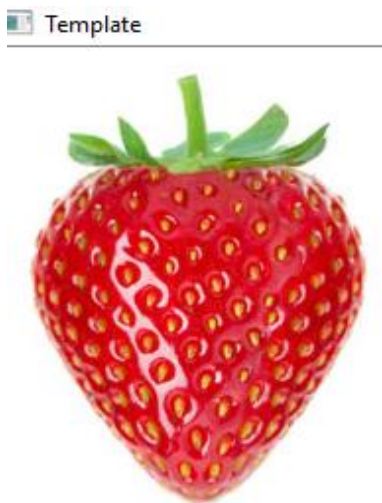

Fig. 11: Template image

IImage

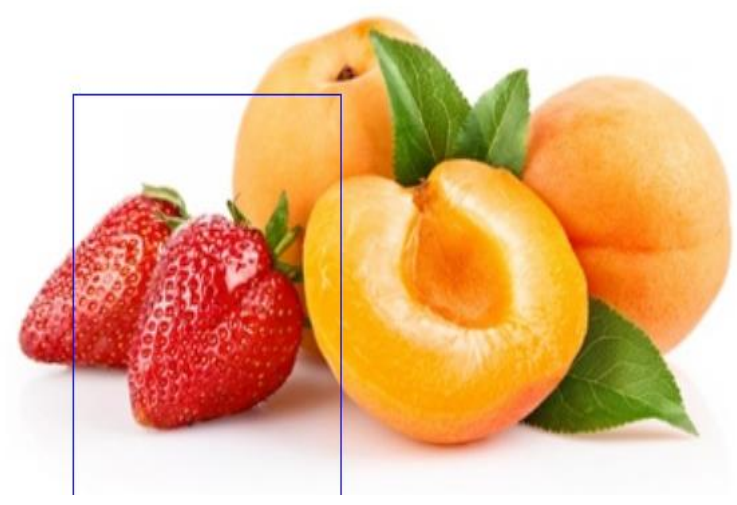

Fig .12: Reference image

\section{Conclusion}

Template matching is the process of identifying a patch of a template image in a given source image. In this paper, we offer a strategy that makes the innocent layout coordinating calculation scale and edge invariant amid the picture acknowledgment process. The proposed calculation handles the self-assertive changes of the picture fix concerning size and edge by a thorough pursuit of all blends of sizes are done alongside different mixes of points. The picture forces are balanced utilizing histogram leveling to upgrade the picture differentiate. These pictures are then subjected to perform layout coordinating utilizing standardized cross relationship to quantify similitude between those two.

\section{References}

[1] Chang Liu, Yongqiang Bai, A new fast and robust template matching with randomness, ISSN: 1948-9447,July 2017

[2] T. Dekel, S. Oron, M. Rubinstein, S. Avidan, and W. T. Freeman. Best-buddies similarity for robust template matching. In Proceedings of the IEEE Conference on Computer Vision and Pattern Recognition, pages 2021-2029, 2015.

[3] M.-S. Choi and W.-Y. Kim. A novel two stage template matching method for rotation and illumination invariance. Pattern recognition, 35(1):119-129, 2002.

[4] Ahmet Burak Yoldemir, Mehmet Sezgin ,Rotation and scale invariant template matching applied to buried object discrimination in gpr data, December 2010

[5] Lowe D G. Distinctive image features from scale-invariant keypoints. International journal of computer vision, 60(2): 91110,2004

[6] M. Gharavi-Alkhansari. A fast globally optimal algorithm for template matching using low-resolution pruning. IEEE Transactions on Image Processing, 10(4):526-533, 2001.

[7] J. Shi and C. Tomasi, "Good Features to Track", Proc. IEEE Conf. on Computer Vision and Pattern Recognition, 1994.

[8] J. P. Lewis,Fast Normalized Cross-Correlation, Industrial Light \& Magi.

[9] T. Padmapriya and V. Saminadan, "Improving Throughput for Downlink Multi user MIMO-LTE Advanced Networks using SINR approximation and Hierarchical CSI feedback", International Journal of Mobile Design Network and InnovationInderscience Publisher, ISSN : 1744-2850 vol. 6, no.1, pp. 1423, May 2015.

[10] S.V.Manikanthan and K.Baskaran "Low Cost VLSI Design Implementation of Sorting Network for ACSFD in Wireless Sensor Network", CiiT International Journal of Programmable Device Circuits and Systems,Print: ISSN 0974 - 973X \& Online: ISSN 0974 - 9624, Issue : November 2011, PDCS112011008

[11] S.V. Manikanthan , T. Padmapriya "An enhanced distributed evolved node-b architecture in 5G tele-communications network" International Journal of Engineering \& Technology (UAE), Vol 7 Issues No (2.8) (2018) 248-254.March2018 